\title{
Multi-morbidity patterns in people with HIV
}

Davide De Francesco (1), Caroline A. Sabin (1) and Peter Reiss (2)

(1) Institute for Global Health, University College London, London, UK;

(2) Department of Global Health, Amsterdam University Medical Centers, location AMC, and Amsterdam Institute for Global Health and Development, Amsterdam, The Netherlands

\section{Corresponding author}

Mr. Davide De Francesco

Institute for Global Health, UCL, Royal Free Campus, Rowland Hill Street, London, NW3 2PF.

Email: d.defrancesco@ucl.ac.uk

Telephone: +44 2077940500 ext. 38827 


\section{Abstract}

Purpose of review: With the progressive ageing of populations of people with HIV (PWH), multimorbidity is increasing. Multi-morbidity patterns, i.e. groups of comorbidities that are likely to cooccur, may suggest shared aetiologies or common risk factors. We review the literature regarding multi-morbidity patterns identified with data-driven approaches and discuss the methodology and the potential implications of the findings.

Recent findings: Despite the substantial heterogeneity in the methods used to identify multimorbidity patterns, patterns of mental health problems, cardiovascular diseases, metabolic disorders and musculoskeletal problems are consistently reported in the general population, with patterns of mental health problems, cardiovascular diseases or metabolic disorders commonly reported in PWH. In addition to these, patterns of lifestyle-related comorbidities such as sexually transmitted diseases, substance use (alcohol, recreational drugs, tobacco smoking) or their complications, seem to occur among PWH.

Summary: Multi-morbidity patterns could inform the development of appropriate guidelines for the prevention, monitoring and management of multiple comorbidities in $\mathrm{PWH}$. They can also help to generate new hypotheses on the aetiology underlying previously known and unknown associations between comorbidities and facilitate the identification of risk factors and biomarkers for specific patterns.

Keywords: HIV; Multi-morbidity; multi-morbidity patterns; comorbidity; 


\section{Introduction}

Multi-morbidity has been defined as "the co-existence of two or more chronic conditions, each one of which is either a physical non-communicable disease, a mental health condition or an infectious disease" (1). In the context of people with HIV (PWH), this definition can be adapted to refer to the co-existence of two or more additional chronic conditions in an individual.

With the widespread use of combination antiretroviral treatment (cART) and the consequent improvement in survival of PWH $(2,3)$, an increasing number of PWH are reaching the age at which multi-morbidity becomes more likely. As several co-morbidities have been reported to occur at an earlier age in PWH than in HIV-negative individuals (4-7), multi-morbidity has become an increasing focus of research in HIV settings because of its increasing prevalence (8) and its negative association with health outcomes (9-11). The clinical management of individuals with multiple comorbidities is typically more challenging than that of individuals with a single condition $(12,13)$ with most treatments and practice guidelines targeting single diseases independently, and lacking guidance on how to address the complex needs of individuals with multi-morbidity.

Although any combination of co-morbidities is, in theory, possible, there is evidence to suggest that certain comorbidities are more likely to co-occur within the same individual (14). As clustered comorbidities may be indicative of shared aetiologies or common risk factors, the identification of the most common patterns of comorbidities is recognised as a research priority for both the general population and $\mathrm{PWH}$, in order to improve the delivery of targeted healthcare $(1,15)$.

The number of potential patterns of comorbidities increases exponentially as the number of comorbidities considered increases. For example, a set of 20 comorbidities will result in 380 possible pairs of comorbidities, 1140 triplets, and so on. Even in the absence of a shared aetiology (i.e. comorbidities that develop independently), co-occurrence may still occur simply due to chance. Modern statistical methods have been applied to separate this random co-occurrence from non- 
random co-occurrence of comorbidities and to explore the underlying structure in the distribution of co-occurring comorbidities.

Here we summarise the current evidence regarding the most common patterns of comorbidities, identified with data-driven approaches, both in the general population and in $\mathrm{PWH}$, discussing the methods used and the potential implications of these findings.

\section{An overview of statistical methods}

As highlighted by two systematic reviews, there is considerable variability in the methods used to study associations among and patterns between comorbidities $(16,17)$. The choice of study population, data source, number and type of co-morbidities considered, and statistical methodology will all influence the patterns identified. Whilst most studies have focused on older age groups where multi-morbidity is more likely, fewer studies have included broader age ranges. Study settings include primarily the general population or primary care $(16,17)$, but also confinement $(18)$, a veteran regional network $(19,20)$ and claim-based records from an health insurance company $(21)$. Data sources and methods used to ascertain the presence of a given disease or clinical condition varied considerably across studies; these included one or a combination of participant self-report (either via interview or questionnaire), clinical examination, administrative and clinical health records. Partly as a reflection of the data source, the number of diseases considered ranged from 10 (22) to 263 (23).

Researchers commonly aim to identify the most prevalent combinations (pairs, triplets, quartets, etc.) of co-occurring comorbidities. However, the prevalence of a combination of comorbidities is strongly influenced by the prevalences of the individual comorbidities in question and, therefore, a high prevalence of a particular combination does not, by itself, provide evidence to support a non-random pattern. In order to account for this random co-occurrence, some studies have compared the observed prevalence of distinct comorbidity patterns to the prevalence that would be expected due to chance alone $(21,24,25)$, calculating the observed-to-expected prevalence ratio of each pattern and retaining those with the most extreme values of this ratio (Figure $1 \mathrm{~A}$ ). 
Other statistical approaches with no a priori hypothesis about the possible patterns and that go beyond the random chance of co-occurrence include cluster analysis, principal component analysis (PCA) and exploratory factor analysis (EFA). Cluster analysis is a widely-used statistical method for identifying homogenous distinct groups of objects ('clusters'). The goal of cluster analysis is to find groups of observations (i.e. individuals or comorbidities) such that observations within each group are similar to one another with respect to variables or attributes of interest, whereas observations from different groups are as dissimilar as possible (26) (Figure 1B). While a few studies have followed the classical approach of clustering observations (i.e. study participants) based on some similarity/dissimilarity index derived from a set of variables (i.e. presence/absence of comorbidities) $(19,27-29)$, the desire to find patterns of comorbidities based on their degree of co-occurrence in the same individual may be better addressed by a cluster analysis of variables rather than observations (i.e. co-morbidities rather than participants), and the majority of studies have followed this approach $(16,17)$. The analytical choices made result in several algorithms to perform cluster analysis, the choice of which may ultimately impact the overall result.

PCA and EFA are conceptually-different but mathematically-similar approaches (Figure 1C). Both are data reduction techniques that aim to reduce the size of a large dataset of many interrelated variables and involve the same steps: factoring the correlation or covariance matrix that measures the pairwise associations between variables, extraction of a smaller set of factors/components obtained as combinations of the original variables, rotation of the factors/components to improve interpretability, and selection of the optimal number of factors/components that should be retained. $(30,31)$. However, whilst PCA identifies combinations (components) that maximise the total variance retained in the dataset, EFA identifies combinations (factors) that maximise the covariance explained among the original set of variables. EFA therefore does not attempt to reproduce the intrinsic variance in each variable which is due to measurement error and is not shared with the other variables. Differences exist between the measures used to assess the correlation between comorbidities, rotation techniques and the criteria used to determine the optimal number of components/factors, 
which can translate into different findings. For example, rotation techniques rearrange the coefficients of the original variables in the composition of the components/factors so that, for each component/factor, only a few variables have large coefficients with the remaining variables having small coefficients. This means that the component/factor can be more easily interpreted, whilst the amount of information explained by the rotated components/factors remains unchanged. Two classes of rotation techniques exist: so-called orthogonal rotation techniques, which rotate components/factors in a way so that they are independent to each other, and oblique rotations, which allow components/factors to be correlated. When applied to comorbidity data, each component/factor translates into a multi-morbidity pattern: orthogonal rotations would lead to patterns that are independent and where co-occurrence of different patterns will be unlikely; oblique rotations would allow the possibility of multiple patterns being present in the same individual.

Other methods that have been proposed include multiple correspondence analysis $(32,33)$, association rule analysis (34) and an asymmetric version of the Somers' D statistic (a measure of agreement between categorical variables) for binary data to measure non-random associations between pairs of comorbidities together with a three-step clustering algorithm that enables comorbidities to overlap in more than one cluster (35).

All approaches have their strengths and limitations. For example the observed-to-expected prevalence ratio is affected by technical difficulties associated with the large number of theoreticallypossible combinations of comorbidities. Cluster analysis is sensitive to the choice of the algorithm (36) and does not allow comorbidities to be part of multiple patterns, unless subjects, rather than comorbidities, are grouped. Factor analysis was originally developed for continuous data and it remains unclear how this method adapts to the dichotomous nature of comorbidity data. Moreover, the interpretation of patterns may be difficult when negative weights are assigned to a comorbidity suggesting a negative association between that comorbidity and the others in the same pattern. A systematic review has described the methodological features of 14 studies and found differences in 
both the number of patterns and the number of diseases within each pattern across the three most common approaches (i.e. cluster analysis, PCA/EFA and observed-to-expected prevalence ratio) (16). $\mathrm{Ng}$ et al. (17) applied five analytical methods to the same multi-morbidity data and found that different methods resulted in different multi-morbidity patterns. Similarly, in another study, despite some overlap, there were differences in the patterns identified with hierarchical cluster analysis and EFA (23). It is therefore important that future studies of multi-morbidity patterns in PWH, take into consideration the intrinsic characteristics of the technique used when interpreting the findings.

\section{What is known about multi-morbidity patterns in the general population?}

Considering this heterogeneity in the methods, a comparison of patterns identified across different studies can be challenging. Nevertheless, mental health problems, cardiovascular diseases, metabolic disorders and musculoskeletal problems often feature among the patterns identified in the general population $(16,37,38)$. Reported patterns of mental health problems predominantly included anxiety and depression with some studies reporting associations with other comorbidities such as neurological problems, pain and somatic disorders, substance use, asthma/chronic obstructive pulmonary disease, gastro-intestinal and musculoskeletal disorders $(16,38)$. Patterns of cardiovascular diseases and metabolic disorders have also been frequently reported, with some studies reporting them as separate patterns, others only reporting one or other of the two patterns, and others reporting a single pattern including both cardiovascular diseases and metabolic disorders. Within this cardio-metabolic pattern, the most frequent comorbidities are diabetes, hypertension, heart disease, obesity and hyperlipidaemia. Few studies have reported co-occurrence of either cardiovascular or metabolic disorders with respiratory problems.

Another pattern consistently identified is musculoskeletal disorders (at least one of osteoarthritis, back pain, osteoporosis and arthrosis). Often, this pattern also includes one or more non- 
musculoskeletal disorders including obesity, prostatic hypertrophy, hypertension, gastro-intestinal disorders, respiratory problems, visual or hearing impairment $(16,38)$.

\section{Are multi-morbidity patterns different in $\mathrm{PWH}$ ?}

To our knowledge, only three studies have investigated potential non-random patterns of co-occurring comorbidities in PWH using a statistical approach without any a priori hypothesis, with one study exploring patterns in two separate cohorts (Table 1) (39-41). Using EFA, Goulet et al. (39) identified three different patterns from an original set of 11 individual comorbidities in a cohort of HIV-positive veterans in care in the US. The authors identified a 'mental disorders' pattern including psychiatric disorders, alcohol and drug abuse, a 'medical disorders' pattern with diabetes, hypertension, congestive heart failure and coronary artery disease and an 'alcohol-related complications' pattern of pancreatitis, anaemia and alcohol abuse. Using a similar approach, Kim et al. (40) investigated the distribution in clusters of 15 different comorbidities in PWH in the South-Eastern US. They reported three different patterns: a 'Metabolic' pattern which included conditions such as hypertension, diabetes mellitus and chronic kidney disease; a 'Behavioural' pattern of mood disorders, dyslipidaemia, chronic obstructive pulmonary disease, peptic ulcer disease, osteoarthritis and cardiac disorders; and an additional pattern labelled 'Substance use' consisting of alcohol, substance and tobacco abuse and HCV. More recently, we have reported frequent patterns of co-occurring comorbidities in PWH enrolled in the $\mathrm{AGE}_{\mathrm{h}} \mathrm{IV}$ (The Netherlands) and Pharmacokinetic Observations of PeoPle over fifty (POPPY) study (UK/Ireland) cohorts (41), most of whom were on CART and virally suppressed. Exploring between 42 and 65 individual comorbidities, we found patterns of cardiovascular diseases, metabolic disorders, sexually transmitted diseases, and mental health problems in both cohorts.

Notably, all three published studies of multi-morbidity patterns in PWH reported patterns that are generally reported in the general population (16), such as the ones consisting of mental health problems, cardiovascular diseases or metabolic disorders. In addition to these patterns, other novel 
patterns emerged, including patterns of sexually transmitted diseases, and of conditions related to substance use (alcohol, recreational drugs, tobacco) or their complications. In some way, these are all interrelated with lifestyle and behavioural factors, including the sexually transmitted disease pattern which is likely to reflect exposure to the same common sexual behaviours. These lifestyle-related patterns are expected to be particularly frequent in PWH (compared to the general population) given the higher prevalence of substance use and risk-taking sexual behaviours often reported among populations of PWH (42-45).

Whilst studies focused on both PWH and the general population have been primarily undertaken in resource-rich countries, multi-morbidity patterns in low- and middle-income countries, where HIV and other chronic infectious diseases (e.g. tuberculosis, hepatitis C virus (HCV)) are typically more prevalent (46), are less well documented. For example, sub-Saharan Africa, one of the regions of the world with the highest prevalence of HIV, tuberculosis and HCV (46), has observed a recent rise in morbidity and mortality from non-communicable diseases (47). Whilst chronic infectious and noncommunicable diseases often co-occur $(48,49)$, knowledge about how they distribute in sub-Saharan Africa and other low- and middle-income countries is lacking.

\section{What can we learn from multi-morbidity patterns?}

A thorough understanding of multi-morbidity patterns has important implications for both clinical and research purposes (Figure 2). Multi-morbidity patterns could inform decisions about prevention and monitoring strategies for individuals with one condition aimed at preventing the development of other conditions that may co-occur. For example, based on the finding that depression, anxiety and other mental health problems consistently form a pattern in studies of PWH, the presence of one of these conditions would prompt close monitoring and prevention strategies of the other conditions, whereas treatment of the condition present may also reduce the risk of the others. Ultimately, these datadriven multi-morbidity patterns would provide useful evidence for the development of patient- 
(rather than disease-) oriented guidelines for the management and treatment of individuals with multi-morbidity.

Co-occurring comorbidities may share the same aetiology or exposure to the same risk factors. Therefore, multi-morbidity patterns may indicate previously known pathophysiological mechanisms (e.g. cardiovascular and metabolic disorders) but also suggest previously unknown hypothesisgenerating associations leading to further studies of common underlying pathophysiological pathways. By separating random from non-random co-occurrence of comorbidities, random noise is reduced, allowing researchers to design more powerful studies to identify risk factors or biomarkers for a given pattern, and quantify the impact of patterns on health-related outcomes, quality-of-life and resource use. Subsequent to our identification of patterns in PWH in the UK/Ireland (41), we showed that risk factors and associations with health-related outcomes, functional status and healthcare resource use differed by pattern (50). Knowledge about the modifiable determinants of patterns would provide further evidence to optimizing approaches to prevent multi-morbidity.

\section{Conclusion}

Despite the heterogeneity in the methods used, patterns of cardiovascular and metabolic diseases, mental health problems and musculoskeletal disorders are frequently reported in general population studies. Whilst the cardiovascular and metabolic disease pattern and the mental health patterns were consistently described in cohorts of PWH, other patterns related to lifestyle and behavioural factors have also been reported. Knowledge about such multi-morbidity patterns could help clinicians to deliver targeted treatment and prevention strategies to $\mathrm{PWH}$, and direct future research to understand the mechanisms underlying specific patterns. Future studies should focus on understudied groups of PWH, such as women and PWH in low- and middle-income countries, investigate changes over the life course of multi-morbidity patterns in $\mathrm{PWH}$, and identify the patterns which pose the greatest burden for patients and healthcare systems. 


\section{Key points}

- With the progressive ageing of HIV-positive populations, multi-morbidity is increasing and the study of multi-morbidity patterns, i.e. groups of comorbidities that are likely to co-occur, has been identified as research priority to improve the quality of life of PWH.

- There is great heterogeneity in the methods used to identify multi-morbidity patterns in the general population. The most commonly used statistical methods are observed to expected prevalence ratio, cluster analysis, principal component analysis and exploratory factory analysis.

- Despite the heterogeneity in analytical approaches, patterns of mental health problems, cardiovascular diseases, metabolic disorders and musculoskeletal problems are consistently reported in the general population.

- Three studies have explored multi-morbidity patterns in PWH and have consistently reported patterns of mental health problems, cardiovascular diseases or metabolic disorders, with the addition of patterns of lifestyle-related comorbidities such as sexually transmitted diseases, substance use or their complications.

- Knowledge of frequent multi-morbidity patterns could inform the development of appropriate guidelines for the management of PWH with multi-morbidity. In research settings, multi-morbidity patterns can generate new hypotheses on the aetiology underlying the associations between comorbidities and facilitate studies to identify risk factors and biomarkers for specific patterns. 


\section{Acknowledgements}

None

\section{Financial support and sponsorship}

None

\section{Conflicts of interest}

DDF has no conflicts of interest. CAS. has received funding from Gilead Sciences, ViiV Healthcare, and Janssen-Cilag for membership of data safety and monitoring boards, advisory boards, and speaker panels and for preparation of educational materials. PR has served as a scientific advisor to Bristol-Myers Squibb, Gilead Sciences, Grupo Ferrer, GlaxoSmithKline, Janssen Pharmaceuticals, Merck \& Co, Inc, and ViiV Healthcare. He has served on data and safety monitoring boards and endpoint adjudication committees for Janssen Pharmaceuticals and his institution has received honoraria for speaking engagements at scientific conferences from Bristol-Myers Squibb, Gilead Sciences, Inc, GlaxoSmithKline. He has received research support from Gilead Sciences, ViiV Healthcare, Merck \& Co, Inc, Janssen Pharmaceuticals, Bristol-Myers Squibb, Abbott, and Boehringer Ingelheim Pharmaceuticals.

\section{Figure/Table legend}

\section{Table 1.}

Heading: Summary of methods and findings of studies on multi-morbidity patterns in PWH

\section{Figure 1.}

Heading: Overview of statistical methods. A) Observed to expected ratios of pairs of four hypothetical conditions. B) Cluster analysis: scatter plot of 13 hypothetical conditions indicated by capital letters (left) and output of the cluster analysis showing three patterns. C) PCA/EFA: individuals mapped in a three-dimensional space defined by the likelihood of three hypothetical conditions (left) and in the two-dimensional space defined by the two extracted components/factors (i.e. patterns)

\section{Figure 2.}

Heading: Potential benefits of identifying multi-morbidity patterns 


\section{References}

1. MacMahon S. Multimorbidity: A priority for global health research. The Academy of Medical Sciences: London, UK. 2018.

- This policy report summarises the available evidence on multi-morbidity and identifies key research priorities to understand multi-morbidity in the general population.

2. Palella Jr FJ, Delaney KM, Moorman AC, Loveless MO, Fuhrer J, Satten GA, et al. Declining morbidity and mortality among patients with advanced human immunodeficiency virus infection. New England Journal of Medicine. 1998;338(13):853-60.

3. Mocroft A, Ledergerber B, Katlama C, Kirk O, Reiss P, d, Monforte AdA, et al. Decline in the AIDS and death rates in the EuroSIDA study: an observational study. The Lancet. 2003;362(9377):22-9.

4. Guaraldi G, Orlando G, Zona S, Menozzi M, Carli F, Garlassi E, et al. Premature age-related comorbidities among HIV-infected persons compared with the general population. Clinical Infectious Diseases. 2011:cir627.

5. Schouten J, Wit FW, Stolte IG, Kootstra N, van der Valk M, Geerlings SG, et al. Cross-sectional comparison of the prevalence of age-associated comorbidities and their risk factors between HIV-infected and uninfected individuals: the AGEhIV cohort study. Clinical Infectious Diseases. 2014:ciu701.

6. Desquilbet L, Jacobson LP, Fried LP, Phair JP, Jamieson BD, Holloway M, et al. HIV-1 infection is associated with an earlier occurrence of a phenotype related to frailty. The Journals of Gerontology Series A: Biological Sciences and Medical Sciences. 2007;62(11):1279-86.

7. Rasmussen LD, May MT, Kronborg G, Larsen CS, Pedersen C, Gerstoft J, et al. Time trends for risk of severe age-related diseases in individuals with and without HIV infection in Denmark: a nationwide population-based cohort study. The Lancet HIV. 2015;2(7):e288-e98.

8. Wong C, Gange SJ, Moore RD, Justice AC, Buchacz K, Abraham AG, et al. Multimorbidity among persons living with human immunodeficiency virus in the United States. Clinical Infectious Diseases. 2017;66(8):1230-8.

9. Rodriguez-Penney AT, ludicello JE, Riggs PK, Doyle K, Ellis RJ, Letendre SL, et al. Co-morbidities in persons infected with HIV: increased burden with older age and negative effects on healthrelated quality of life. AIDS patient care and STDs. 2013;27(1):5-16.

10. Morgan EE, ludicello JE, Weber E, Duarte NA, Riggs PK, Delano-Wood L, et al. Synergistic effects of HIV infection and older age on daily functioning. Journal of acquired immune deficiency syndromes (1999). 2012;61(3):341. 
11. Van Duin MJ, Conde R, Wijnen B, Evers SM, Gonzalez-Rodriguez JL, Govers MJ, et al. The impact of comorbidities on costs, utilities and health-related quality of life among HIV patients in a clinical setting in Bogotá. Expert Review of Pharmacoeconomics \& Outcomes Research. 2016:1-8.

12. Fortin M, Soubhi H, Hudon C, Bayliss EA, van den Akker M. Multimorbidity's many challenges. BMJ : British Medical Journal. 2007;334(7602):1016-7.

13. Starfield B. Challenges to primary care from co-and multi-morbidity. Primary health care research \& development. 2011;12(01):1-2.

14. Divo MJ, Martinez CH, Mannino DM. Ageing and the epidemiology of multimorbidity. Eur Respiratory Soc; 2014.

15. High KP, Brennan-Ing M, Clifford DB, Cohen MH, Currier J, Deeks SG, et al. HIV and aging: state of knowledge and areas of critical need for research. A report to the NIH Office of AIDS Research by the HIV and Aging Working Group. Journal of acquired immune deficiency syndromes. 2012;60(Suppl 1):S1-18.

16. Prados-Torres A, Calderón-Larrañaga A, Hancco-Saavedra J, Poblador-Plou B, van den Akker M. Multimorbidity patterns: a systematic review. Journal of Clinical Epidemiology. 2014;67(3):25466.

17. Ng SK, Tawiah R, Sawyer M, Scuffham P. Patterns of multimorbid health conditions: a systematic review of analytical methods and comparison analysis. International journal of epidemiology. 2018;47(5):1687-704.

- This study reviewed the statistical methodology used to identify multi-morbidity patterns by previous studies and applied five different methods to the same dataset, highlighting the differences in the patterns identified by the different methods.

18. Gates ML, Hunter EG, Dicks V, Jessa PN, Walker V, Yoo W. Multimorbidity patterns and associations with functional limitations among an aging population in prison. Archives of gerontology and geriatrics. 2018;77:115-23.

19. Goldstein G, Luther JF, Jacoby AM, Haas GL, Gordon AJ. A taxonomy of medical comorbidity for veterans who are homeless. Journal of Health care for the Poor and Underserved. 2008;19(3):991-1005.

20. Goldstein G, Luther JF, Haas GL, Appelt CJ, Gordon AJ. Factor structure and risk factors for the health status of homeless veterans. Psychiatric quarterly. 2010;81(4):311-23.

21. van den Bussche $H$, Koller D, Kolonko $T$, Hansen $H$, Wegscheider $K$, Glaeske $G$, et al. Which chronic diseases and disease combinations are specific to multimorbidity in the elderly? Results of a claims data based cross-sectional study in Germany. BMC Public Health. 2011;11(1):101. 
22. Islam MM, Valderas JM, Yen L, Dawda P, Jowsey T, McRae IS. Multimorbidity and comorbidity of chronic diseases among the senior Australians: prevalence and patterns. PloS one. 2014;9(1):e83783.

23. Roso-Llorach A, Violán C, Foguet-Boreu Q, Rodriguez-Blanco T, Pons-Vigués $M$, Pujol-Ribera E, et al. Comparative analysis of methods for identifying multimorbidity patterns: a study of 'realworld'data. BMJ open. 2018;8(3):e018986.

24. Wong A, Boshuizen HC, Schellevis FG, Kommer GJ, Polder JJ. Longitudinal administrative data can be used to examine multimorbidity, provided false discoveries are controlled for. Journal of clinical epidemiology. 2011;64(10):1109-17.

25. Freund T, Kunz CU, Ose D, Szecsenyi J, Peters-Klimm F. Patterns of multimorbidity in primary care patients at high risk of future hospitalization. Population Health Management. 2012;15(2):11924.

26. Everitt BS, Landau S, Leese M, Stahl D. An Introduction to Classification and Clustering. Cluster Analysis: John Wiley \& Sons, Ltd; 2011. p. 1-13.

27. Newcomer SR, Steiner JF, Bayliss EA. Identifying subgroups of complex patients with cluster analysis. The American journal of managed care. 2011;17(8):e324-32.

28. Dörenkamp S, Mesters I, Schepers J, Vos R, van den Akker M, Teijink J, et al. Disease combinations associated with physical activity identified: the SMILE cohort study. BioMed research international. 2016;2016.

29. Marengoni A, Roso-Llorach A, Vetrano DL, Fernández-Bertolín S, Guisado-Clavero M, Violán C, et al. Patterns of Multimorbidity in a Population-Based Cohort of Older People: Sociodemographic, Lifestyle, Clinical, and Functional Differences. The Journals of Gerontology: Series A. 2019.

30. Johnson RA, Wichern DW. Applied multivariate statistical analysis: Pearson; 2014.

31. Jolliffe I. Principal component analysis: Wiley Online Library; 2002.

32. García-Olmos L, Salvador CH, Alberquilla Á, Lora D, Carmona M, García-Sagredo P, et al. Comorbidity Patterns in Patients with Chronic Diseases in General Practice. PLOS ONE. 2012;7(2):e32141.

33. Ruiz M, Bottle A, Long S, Aylin P. Multi-Morbidity in Hospitalised Older Patients: Who Are the Complex Elderly? PLOS ONE. 2016;10(12):e0145372.

34. Held FP, Blyth F, Gnjidic D, Hirani V, Naganathan V, Waite LM, et al. Association rules analysis of comorbidity and multimorbidity: The Concord Health and Aging in Men Project. Journals of Gerontology Series A: Biomedical Sciences and Medical Sciences. 2015;71(5):625-31. 
35. Ng SK, Holden L, Sun J. Identifying comorbidity patterns of health conditions via cluster analysis of pairwise concordance statistics. Statistics in Medicine. 2012;31(27):3393-405.

36. Morgan BJ, Ray AP. Non-uniqueness and inversions in cluster analysis. Applied statistics. 1995:117-34.

37. Sinnige J, Braspenning J, Schellevis F, Stirbu-Wagner I, Westert G, Korevaar J. The Prevalence of Disease Clusters in Older Adults with Multiple Chronic Diseases - A Systematic Literature Review. PLOS ONE. 2013;8(11):e79641.

38. Violan C, Foguet-Boreu Q, Flores-Mateo G, Salisbury C, Blom J, Freitag M, et al. Prevalence, Determinants and Patterns of Multimorbidity in Primary Care: A Systematic Review of Observational Studies. PLOS ONE. 2014;9(7):e102149.

39. Goulet JL, Fultz SL, McGinnis KA, Justice AC. Relative prevalence of comorbidities and treatment contraindications in HIV-mono-infected and HIV/HCV-co-infected veterans. Aids. 2005;19:S99S105.

40. Kim DJ, Westfall AO, Chamot E, Willig AL, Mugavero MJ, Ritchie C, et al. Multimorbidity patterns in HIV-infected patients: the role of obesity in chronic disease clustering. Journal of acquired immune deficiency syndromes (1999). 2012;61(5):600.

41. De Francesco D, Verboeket SO, Underwood J, Bagkeris E, Wit FW, Mallon PWG, et al. Patterns of Co-occurring Comorbidities in People Living With HIV. Open Forum Infectious Diseases. 2018;5(11):ofy272-ofy.

- This is the most recent and most comprehensive study of multi-morbidity patterns in PLWH. Patterns of cardiovascular diseases, metabolic disorders, sexually transmitted diseases and mental health problems were consistently found in two independent cohort of PLWH.

42. Mdodo R, Frazier EL, Dube SR, Mattson CL, Sutton MY, Brooks JT, et al. Cigarette smoking prevalence among adults with HIV compared with the general adult population in the United States: cross-sectional surveys. Annals of internal medicine. 2015;162(5):335-44.

43. Garin N, Velasco C, De Pourcq J, Lopez B, Gutierrez MdM, Haro J, et al. Recreational drug use among individuals living with HIV in Europe: review of the prevalence, comparison with the general population and HIV guidelines recommendations. Frontiers in Microbiology. 2015;6(690).

44. Fuster-RuizdeApodaca MJ, Castro-Granell V, Garin N, Laguía A, Jaén Á, Iniesta C, et al. Prevalence and patterns of illicit drug use in people living with HIV in Spain: A cross-sectional study. PLOS ONE. 2019;14(6):e0211252. 
45. Van Kesteren NM, Hospers HJ, Kok G. Sexual risk behavior among HIV-positive men who have sex with men: a literature review. Patient education and counseling. 2007;65(1):5-20.

46. James SL, Abate D, Abate KH, Abay SM, Abbafati C, Abbasi N, et al. Global, regional, and national incidence, prevalence, and years lived with disability for 354 diseases and injuries for 195 countries and territories, 1990-2017: a systematic analysis for the Global Burden of Disease Study 2017. The Lancet. 2018;392(10159):1789-858.

47. Statistics South Africa. Mortality and causes of death in South Africa,. 2016.

48. Oni T, Youngblood E, Boulle A, McGrath N, Wilkinson RJ, Levitt NS. Patterns of HIV, TB, and noncommunicable disease multi-morbidity in peri-urban South Africa- a cross sectional study. BMC Infect Dis. 2015;15:20.

49. Achwoka D, Waruru A, Chen TH, Masamaro K, Ngugi E, Kimani M, et al. Noncommunicable disease burden among HIV patients in care: a national retrospective longitudinal analysis of HIVtreatment outcomes in Kenya, 2003-2013. BMC Public Health. 2019;19(1):372.

50. De Francesco D, Underwood J, Bagkeris E, Anderson J, Williams I, Vera J, et al. Risk factors and impact of patterns of co-occurring comorbidities in people living with HIV. AIDS (London, England). 2019.

$\bullet \bullet$ This is the first study investigating the differential risk factors and impact of multi-morbidity patterns frequently observed in PLWH. 